\title{
HS DIC-system application for strain and displacement measurements under static-dynamic coupling loading
}

\author{
Z.Q. Yin \\ School of Mineral and Safety and Postdoctoral Station of Mining Engineering, Anhui University of Science \\ and Technology, Huainan, Anhui, China
}

X.B. Li

School of Resources and Safety Engineering, Central South University, Changsha, China

Y. Zou

Ecole Polytechnique Federale de Lausanne (EPFL), Laboratory of Rock Mechanics (LMR), CH-1015

Lausanne, Switzerland

\begin{abstract}
To study the deformation and fracture of sandstone under static-dynamic coupled load, a cylindrical specimen under pre-static axial and confining pressure was dynamically loaded using an improved split Hopkinson pressure bar (SHPB). Through the application of a special shape striker, stress equilibrium and nearly constant strain rate in specimen were achieved. During dynamic tests, the failure process of the specimen was completely monitored ( 7 frames at a time resolution of $25 \mu \mathrm{s}$ ) by a high speed (HS) camera. Furthermore, the recorded images were matched with the loading steps through a specified trigger mode, based on which both full-field displacement values and the corresponding surface in-plane strain were obtained via digital image correlation (DIC) system. Finally, analysis on the surface deformation and failure mode of specimen shows that the sample presents an interaction of tension-shear failure and expansion failure under the axial static pressure of $72 \mathrm{MPa}$, which reflects the effect of axial static pressure on the dynamic fracture mode of the sample surface.
\end{abstract}

\section{INTRODUCTION}

For the past few years, with the increasing depth of underground excavation, mechanical behaviors of rock have been observed to be different from previous experiences and are difficult to be explained by traditional theories (Li \& Gong 2010). Actually, in the circumstances of deep rock excavation the surrounding rock is not only under high static stress, but also under the influence of stress unloading owing to the formation of free surface which gives rise to stress state change from original three-dimensional to two-dimensional or one-dimensional stress, as well as facing the blasting or drilling during excavation and other dynamic loads (Li \& Zhou 2008). In the combined action of high stress and dynamic loading, the rock mass in the excavation face is prone to rockburst (Yin \& Li 2012). Therefore, study on the destruction mechanism of high stress rock under dynamic disturbance is of great significance to practical engineering.

The digital image correlation (DIC) method is widely used in experimental mechanics as a practical and effective tool for full-field deformation measurement (Peters \& Ranson 1982). This technique is easy to manipulate and can provide satisfactory resolution of displacement and strain fields (Zhou \& Chen 2011). DIC techniques were used for the quasi-static and high strain rate experiments to obtain the in-plane strain field over the entire specimen surface (Koerber \& Xavier 2010). Pierron \& Sutton (2010) used a high speed camera for the high strain rate experiment, in which specimen deformation was monitored and fields method of a three point bending was analyzed.

In this work, the mechanical properties of sandstone under static-dynamic coupling loading are studied using the SHPB device, and a high speed camera was used to monitor the fracture process of the specimen. Based on the DIC method, the surface deformation and failure mode of specimen were analysed. 


\section{EXPERIMENTS}

\subsection{Sample preparation}

The samples were drilled from the same integral and uniform block of sandstone to ensure the homogeneity. The cylindrical specimen was made with the dimensions of $50 \mathrm{~mm} \times 50 \mathrm{~mm}$, and carefully polished at both ends and lateral side. So, the non-parallelism and the non-perpendicularity are both less than $0.02 \mathrm{~mm}$. The sample is gray and smooth on surface, with no distinct interspace. The density of specimen is $2.50 \mathrm{t} / \mathrm{m}^{3}$. The static uniaxial compressive strength of specimens is approximately $90 \mathrm{MPa}$.

\subsection{Experimental setup}

The improved SHPB test system can be used for impact tests under triaxial pre-pressure, as shown in Figure 1. The stress transmission component is made up of two long elastic bars (input bar, output bar). The elastic bars are $2 \mathrm{~m}$ in length and $50 \mathrm{~mm}$ in diameter. The specimen is sandwiched between the two elastic bars. Strain gauges are glued on the surface of the middle of elastic bars to measure strain histories induced by the stress waves propagating along the elastic bars. The material quality of the bullet and the maximum diameter of the projectile body are the same as those of the input bar and output bar. A conical bullet is used in the improved test system to eliminate the oscillation, and obtain a stable half sine wave loading. The stress waves recorded with a CS-1D super dynamic meter and DL 750 oscilloscope (Yokogawa).The confining pressure and static axial pressure are manually loaded. There is a hydraulic valve at the confining pressure and static axial stress loading equipment to control the stress unloading velocity by manually adjusting the valve opening level.

Specimen stress $\left(\sigma_{S}\right)$, strain $\left(\varepsilon_{S}\right)$ and strain rate $\left(\dot{\varepsilon}_{S}\right)$ are calculated from the following equations:

$$
\begin{aligned}
& \sigma_{S}=E_{B a r} \frac{A_{B a r}}{A_{S}} \varepsilon_{t} \\
& \varepsilon_{S}=-2 \frac{C_{0}}{L_{S}} \int_{0}^{t} \varepsilon_{t} d t \\
& \dot{\varepsilon}_{S}=-2 \frac{C_{0}}{L_{S}} \varepsilon_{r}
\end{aligned}
$$

Where $C_{0}$ is the elastic wave speed in bars, and $A_{B a r}$ and $A_{s}$ are the cross-section areas of the bar and specimen, respectively. $\varepsilon_{t}$ and $\varepsilon_{r}$ are the transmitted and reflected strains, respectively. $L_{S}$ is the specimen length.

In the present experiment, photographs of the specimen were taken using a PHOTRON FASTCAM SA1.1 high speed camera, coupled with a PALLITE high strength and no stroboscopic light source and positioned at $0.7 \mathrm{~m}$ away from the specimen surface. The frame-rate of 40,000 fps with an image resolution of 448 pixels $\times 288$ pixels and a shutter speed of $1 \mu \mathrm{s}$ was selected. The specimen was speckled with black and white paint as show in Figure 2.

A triggering system is composed of strain gauges (which one is on the surface of the input bar) and oscilloscope. When the bullet hits the input bar, the stress wave is produced and propagating in the input bar. A TTL electrical signal which can be used for triggering will be generated when the oscilloscope recording this stress wave signal by strain gauges.

To match the recorded images with the loading steps, delay time from the loading start time to the triggering start time has been to determined to be $218 \mu \mathrm{s}$, based on the combined consideration of the travel time from strain gauge to the specimen end (wave velocity $5410 \mathrm{~m} / \mathrm{s}$, distance $1.49 \mathrm{~m}$ ) and the pre trigger time $57 \mu$ shich is show in Figure 3. 


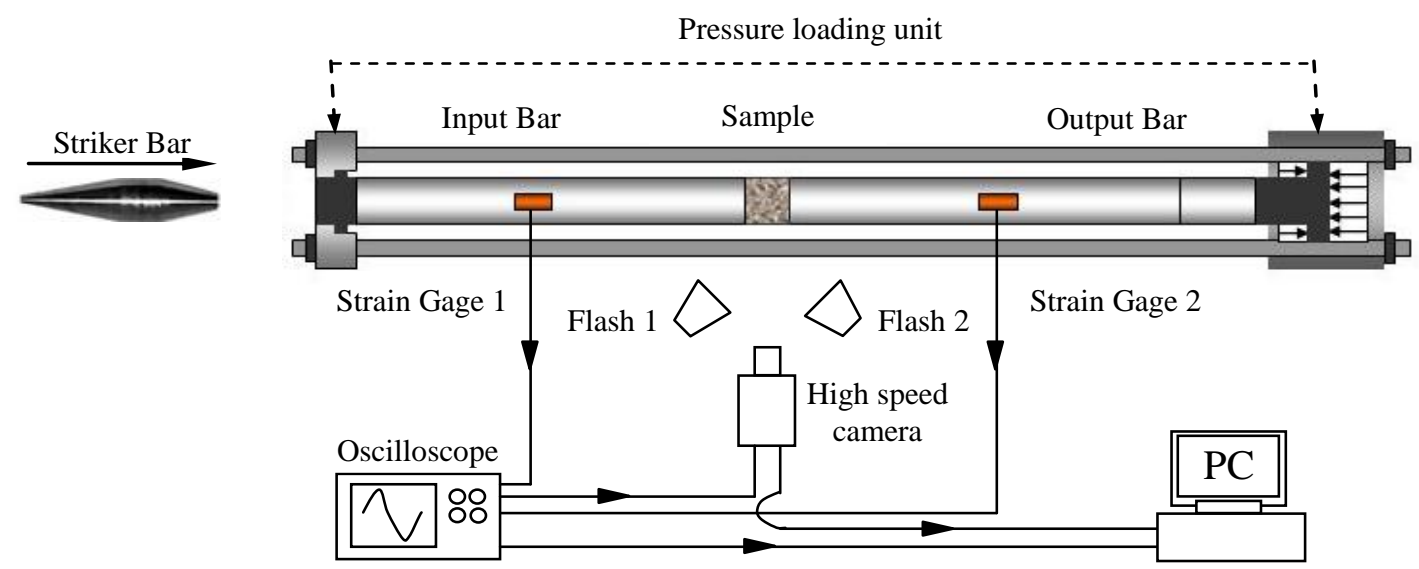

Figure 1. Geometry of the static-dynamic coupling loading test (Gong \& Li 2010).

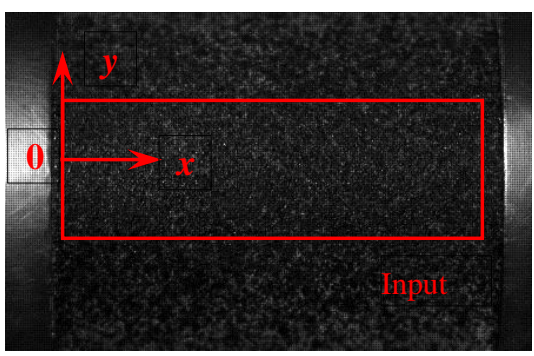

Figure 2. Schematic of the test specimen.

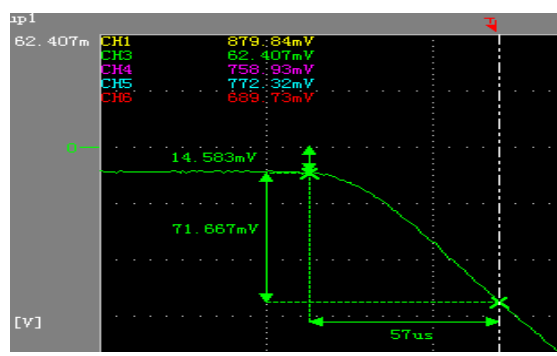

Figure 3. The pre trigger time measurement.

\subsection{Principle of the digital image correlation}

DIC operates through the mathematical comparison of two sub-images from a larger pair of displaced images. The primary assumption of DIC is that the essential characteristics of the speckle pattern remain the same before and after deformation to determine the distortion of a small region. This method uses the correlation coefficient $C$ as a description parameter (Ma \& Jin 2002):

$$
C(u, v)=\frac{\sum_{y=1}^{y=n} \sum_{x=1}^{x=m} f\left(x_{i}, y_{i}\right) \cdot g\left(x_{i}, y_{i}\right)}{\sqrt{\sum_{y=1}^{y=n} \sum_{x=1}^{x=m} f\left(x_{i}, y_{i}\right)^{2} \cdot \sum_{y=1}^{y=n} \sum_{x=1}^{x=m} g\left(x_{i}, y_{i}\right)^{2}}}
$$

where $\left(x_{i}, y_{i}\right)$ are the Cartesian coordinates of the resource and target points located in the reference and deformed images, respectively; $f\left(x_{i}, y_{i}\right)$ and $g\left(x_{i}, y_{i}\right)$ are the grey values of the subset in the non-deformed and deformed images, respectively.

The magnitude of correlation coefficient $C$ varies from 0 to 1 , with 1 signifying a perfect match between the two images. When deformation occurs on the specimen surface in a small region, local characteristic of the speckle images is changed, and the correlation coefficient becomes smeller than other areas where no apparent deformation happens. In this paper, the value of $C$ is much more than 0.95 in the area of interest, indicating that the two speckle images captured before and after a deformation match each other well.

\section{RESULTS AND DISCUSSION}

Dynamic photographs, which can indicate the surface deformation process and crack 
propagation in sample, were obtained by the high-speed camera. Figure 4 shows 6 representative images of this process which were also marked at the corresponding point on the stress-time curve.
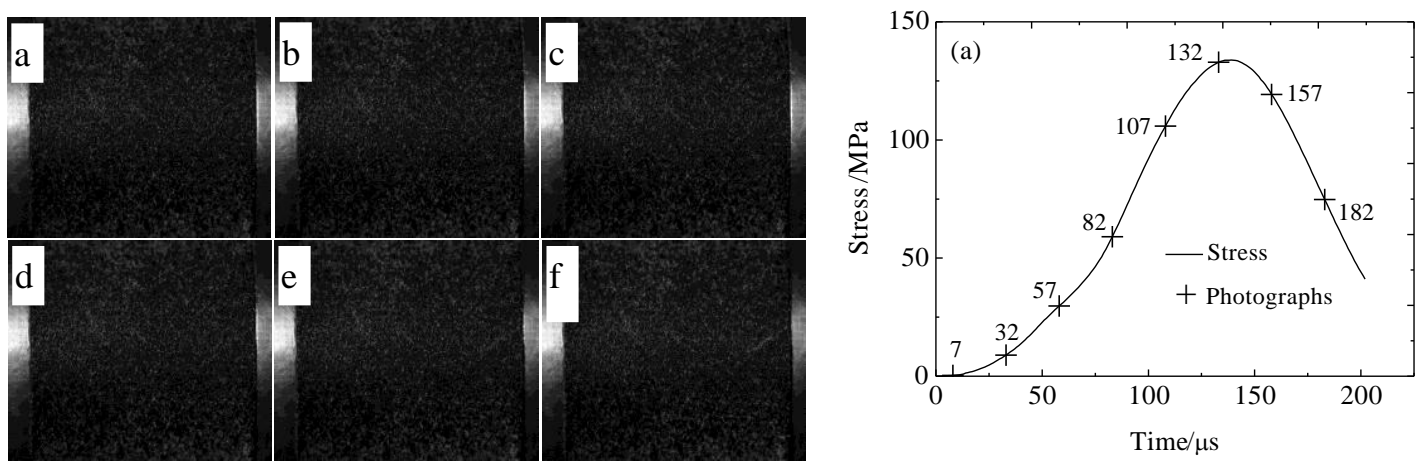

Figure 4. Failure patterns for specimen and stress-time history with high speed images (a) $\mathrm{t}=7$;(b) $\mathrm{t}=32 \mu \mathrm{s}$;(c) $\mathrm{t}=57 \mu \mathrm{s}$; (d) $\mathrm{t}=82 \mu \mathrm{s}$;(e) $\mathrm{t}=107 \mu \mathrm{s}$;(f) $\mathrm{t}=132 \mu \mathrm{s}$.

The field of speckle pattern was depicted by red line in Figure 2 covering about 360 pixels $\times 140$ pixels and being $2 \mathrm{~mm}$ away from both two ends of specimen in order to ensure the correlation. This specified region (box in red) was analyzed by SH DIC to match with the initial image recorded before deformation. The displacement distributions are shown in Figures 5 and 6.
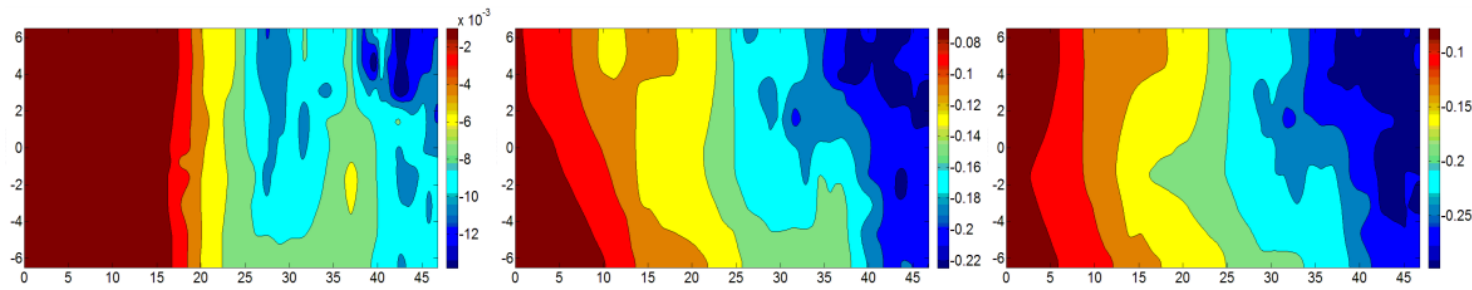

Figure 5. $u_{x}$ displacement, in mm (axes as in Fig. 2): (a) $t=7 \mu \mathrm{s}$; (d) $t=82 \mu \mathrm{s}$; (f) $t=132 \mu \mathrm{s}$.
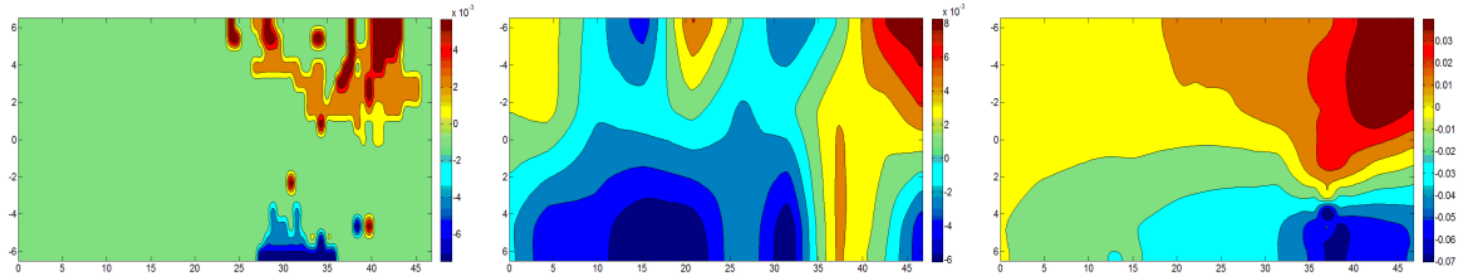

Figure $6 u_{y}$ displacement, in mm (axes as in Fig. 2): (a) $t=7 \mu \mathrm{s}$; (d) $t=82 \mu \mathrm{s}$; (f) $t=132 \mu \mathrm{s}$.

Figure 5 (a) shows that at $7 \mu \mathrm{s}$, the displacement at the left end of specimen is zero. The time of stress wave propagating through the specimen is about $14 \mu \mathrm{s}$. So, at $7 \mu \mathrm{s}$, the stress wave just arrives at the middle of the sample. The displacement of specimen at $7 \mu$ s demonstrates that the time precision of HS DIC system is on microsecond level.

The circumferential expansion characteristic of sample is unfolded by $u_{y}$ displacement. Figure 6 reveals the heterogeneity of circumferential expansion in specimen during the static-dynamic coupling loading. It can be seen that the volume of circumferential expansion at regions close to the input bar is more than that at the counterpart near the output bar.

From the displacement maps, the strains $\varepsilon_{x}$ have been obtained directly from the local second order polynomial fit and are represented in Figure 7. In order to have clearer view of the $\varepsilon_{x}$ strains, the average of $\varepsilon_{x}$ is plotted as function of time. Averages have been calculated over the third of the width on right (close to the input bar), mid and left (close to the output bar). The averages of the $\varepsilon_{x}$ field are shown in Figure 8 (a).The strain averages are plotted in Figure 8 (b).

In order to achieve a convenient comparison of the strains at different positions in sample, the strain-time history induced by the SHPB test and HS DIC are drawn together in Figure 8 (b). It can be found that both values are very close and increase with time before $82 \mu \mathrm{s}$. After $82 \mu \mathrm{s}$ the 
difference in strain values between SHPB test and HS DIC increases, but the strain changing trend between SHPB test and the left end of sample (close to the output bar) is opposite (after $107 \mu \mathrm{s})$. This phenomenon can basically reflect that the left end of sample is mainly destroyed by shear failure.

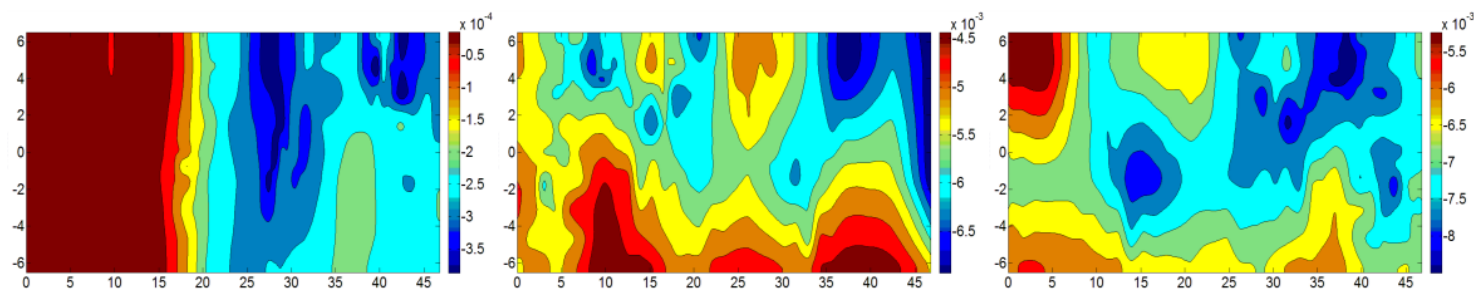

Figure 7. $\varepsilon_{x}$ strains (axes as in Fig. 2): (a) $t=7 \mu \mathrm{s}$; (d) $t=82 \mu \mathrm{s}$; (f) $t=132 \mu \mathrm{s}$.
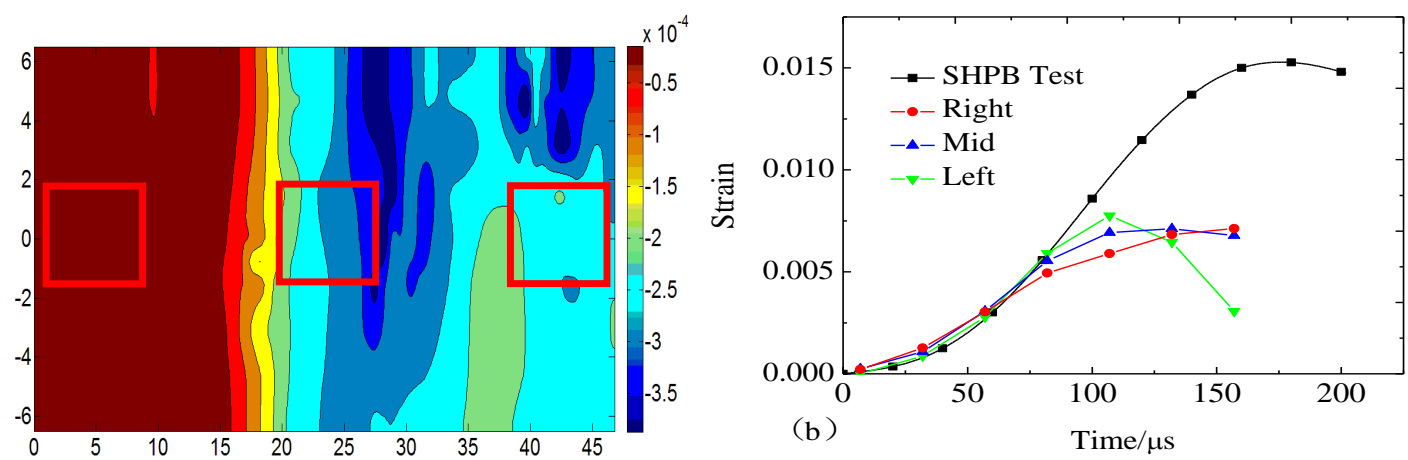

(a) Areas for $\varepsilon_{x}$ averaging

(b) Average $\varepsilon_{x}$ over right and mid and left skins Figure 8. Average strains as a function of time (compressive strain multiplied by -1).

\section{CONCLUSIONS}

In this work, the method combining a static-dynamic coupling loading with the HS DIC technique was successfully used to study the dynamic fracture behavior of sandstone. In situ images of the surface of sandstone in static-dynamic coupling loading test were acquired during dynamic loading step. The displacement and strain fields were measured using the DIC. From the 6 images of the static-dynamic coupling loading impact test, it was possible to derive displacement by image correlation and strain by either spatial and/or temporal differentiation. The deformation and fracture behavior of sandstone were analyzed. It can be found that the failure mode possesses its distinct characteristics under static-dynamic coupling loading. The sample is mainly destroyed by the combined action of tensile expansion failure and tensile shear failure under static-dynamic coupling loading.

\section{ACKNOWLEDGEMENTS}

This work was financially supported by the National Natural Science Foundation of China (50934006), National Basic Research (973) Program of China (2010CB732004) and Scientific Research Fund for Young Teachers of Anhui University of Science and Technology (2012QNY39).

\section{REFERENCES}

Li, X.B. \& Gong, F.Q. 2010. Test study of impact failure of rock subjected to one-dimensional coupled static and dynamic loads. Chinese Journal of Rock Mechanics and Engineering 29(2): 251-260.

Li, X.B. \& Zhou, Z.L. 2008. Innovative testing technique of rock subjected to coupled static and dynamic 
loads. International Journal of Rock Mechanics and Mining Science 45(5): 739-748.

Yin, Z.Q. \& Li, X.B. 2012. Failure characteristics of high stress rock induced by impact disturbance under confining pressure unloading. Transactions of Nonferrous Metals Society of China 22(1): 175184.

Peters, W.H. \& Ranson, W.F. 1982. Digital imaging techniques in experimental stress analysis. Optical Engineering 21(3): 427-31.

Zhou, Z.B. \& Chen, P.W. 2011. Experimental study on the micromechanical behavior of a PBX simulant using SEM and digital image correlation method. Optics and Lasers in Engineering 49(3): 366-371.

Koerber, H. \& Xavier, J. 2010. High strain rate characterisation of unidirectional carbon-epoxy IM7-8552 in transverse compression and in-plane shear using digital image correlation. Mechanics of Materials 42(11): 1004-1019.

Pierron, F. \& Sutton, M.A. 2010. Ultra high speed DIC and virtual fields method analysis of a three point bending impact test on an aluminium bar. Experimental Mechanics 51(4): 537-563.

Gong, F.Q. \& Li, X.B. 2010. Experimental study of dynamic characteristics of sandstone under one-dimensional coupled static and dynamic loads. Chinese Journal of Rock Mechanics and Engineering 29(10): 2076-2085.

Ma S.P. \& Jin, G.C. 2002. Deformation measurement method for rock materials based on natural speckle pattern. Chinese Journal of Rock Mechanics and Engineering 21(6): 792-6. 